\title{
Comparing Self Monitoring Blood Glucose Devices and Laboratory Tests: Over 25 Years Experience
}

\author{
Yuko Harada ${ }^{1}$, Keiichi Harada ${ }^{1}$, Paul Chin Jr. ${ }^{1}$ \\ 1. Internal Medicine, Harada Naika Clinic, Kawasaki, JPN
}

Corresponding author: Yuko Harada, adayuko1219@yahoo.co.jp

\begin{abstract}
Self-monitoring blood glucose (SMBG) devices have been widely used in medical practice for decades. However, there are certain gaps between SMBG device readings and venous blood glucose levels. Here, 3,532 measurement data points were recorded over 25 years to compare SMBG device readings and venous blood glucose levels. The mean absolute difference (MAD) or the GAP was $10.9 \mathrm{mg} / \mathrm{dL}$, and the mean absolute relative difference (MARD) was 8.3\%. The absolute relative difference (ARD) (\%) and absolute difference (AD or GAP) (mg/dL) coefficients of variation (CV) (\%) of 100\% indicate high variance between the capillary BG and venous true $B G$ data. There was a slight skewing of MAD and MARD to the lower body mass index (BMI) side, the higher age side, and the female gender side. There were 41 data points that showed unacceptable gaps of over $50 \mathrm{mg} / \mathrm{dL}$. Such large differences may cause incorrect medications or treatments. All healthcare providers should be aware of the gap between SMBG device readings and venous blood glucose levels.
\end{abstract}

Categories: Endocrinology/Diabetes/Metabolism, Internal Medicine

Keywords: smbg, capillary blood

\section{Introduction}

Self-monitoring blood glucose (SMBG) has been recommended to be routinely used for successful diabetes management and therapy in international guidelines [1-2]. The use of electrode systems to measure blood glucose was advocated by Clark and Lyons in 1962, and the first glucometer (SMBG device) was manufactured in 1986 which was introduced in Japan in 1991 [3-4]. It is known that there are certain gaps between the readings of glucometer and actual blood glucose levels. However, the prompt result of glucometer is more convenient than laboratory testing, which requires a long time. Thus, SMBG devices have been widely used in medical practice, from patients' homes to hospital emergency rooms.

Received 10/23/2019 Review began 11/09/2019 Review ended 11/27/2019 Published 12/01/2019

\section{() Copyright 2019}

Harada et al. This is an open access article distributed under the terms of the Creative Commons Attribution License CC-BY 3.0., which permits unrestricted use, distribution, and reproduction in any medium, provided the original author and source are credited.
In medical office practice, laboratory test results usually take several days because the samples are sent to laboratory agencies. The use of glucometer is the only way to immediately measure the patients' glucose level in medical offices. Over 25 years ago in a private medical clinic, a noted hematologist started to measure blood glucose level simultaneously by both SMBG device and venous blood test, because he believed the capillary blood glucose (local blood sugar) measured by SMBG device should be different from the venous glucose level (systemic blood sugar).

Here we report the extensive analyses of the data from a retrospective study comparing glucometer and laboratory tests. This is the largest and the most extensive database and analyses comparing capillary blood SMBG device measurements and venous blood glucose laboratory testing.

\section{Materials And Methods}

From 1993 through 2019, 5,300 patients visited this general internal medicine clinic. Since the doctors were not endocrinologists, many of the patients were non-diabetic. All of the diabetic patients had type 2 diabetes, and there were several insulin users.

3,532 measurement data points for blood glucose (BG) were recorded and compiled from 81 diabetes patients over a period of over 25 years. In order to minimize human error in measuring blood sugar, trained nurses and doctors performed the capillary finger pricks and blood analyses with self-monitoring blood glucose (SMBG) device, and immediately drew venous blood from the patients' forearms. The nurses and doctors carefully followed the instruction manuals for each device utilized. All the fingers and forearms were disinfected with alcohol before taking samples. The venous blood samples were immediately taken to major blood test laboratories in order to measure blood glucose levels (True BG) and hemoglobin A1C (HbA1c). During the 25 years, five SMBG devices were utilized: one SMBG device for each of three brands, and two generations of SMBG devices for the fourth brand (Device M). 


\section{Cureus}

These data were parameterized according to capillary BG vs. True BG, AD (GAP) (mg/dL) distribution, capillary BG vs. True BG (mg/dL) AD (GAP) - $50 \mathrm{mg} / \mathrm{dL}$ and over, glucometer, body mass Index (BMI) $\left(\mathrm{kg} / \mathrm{m}^{2}\right)$, age, and gender.

Absolute relative difference (ARD) (\%), mean ARD (MARD) (\%), ARD coefficient of variation (CV) (\%), and absolute difference (AD) (the GAP) (mg/dL), mean $\mathrm{AD}(\mathrm{MAD})(\mathrm{mg} / \mathrm{dL})$, and $\mathrm{AD}$ coefficient of variation (CV) (\%) were calculated.

ARD (\%) is the absolute value of the difference between SMBG device measurements (capillary BG) and True BG divided by True BG. MARD (\%) is the mean of the ARDs. ARD coefficient of variation (CV) (\%) is the standard deviation of the ARDs divided by the average of the ARDs. $A D(\mathrm{mg} / \mathrm{dL})$ is the absolute value of the difference between capillary BG and True BG. MAD (mg/dL) is the mean of the ADs. AD coefficient of variation (CV) (\%) is the standard deviation of the ADs divided by the average of the ADs.

Data tables and their corresponding charts are displayed below.

\section{Results}

\section{Capillary blood glucose vs. true blood glucose distribution}

The data measurements were recorded in each patient's chart. Then the data were aggregated and analyzed retrospectively. The data shows SMBG device measurements underestimated true blood glucose (BG) $39 \%$ of the time, matched $4 \%$ of the time and over-estimated true BG $57 \%$ of the time. This distribution is displayed in the data table and chart shown below in Table 1 and Figure 1 .

\begin{tabular}{|l|l|}
\hline Observations: $\mathbf{3 , 5 3 2}$ data points & $39 \%$ \\
\hline Capillary BG $<$ True BG & $4 \%$ \\
Capillary BG $=$ True BG & $57 \%$ \\
\hline Capillary BG $>$ True BG & $4 \%$ \\
\hline
\end{tabular}

TABLE 1: Capillary blood glucose vs. true blood glucose distribution

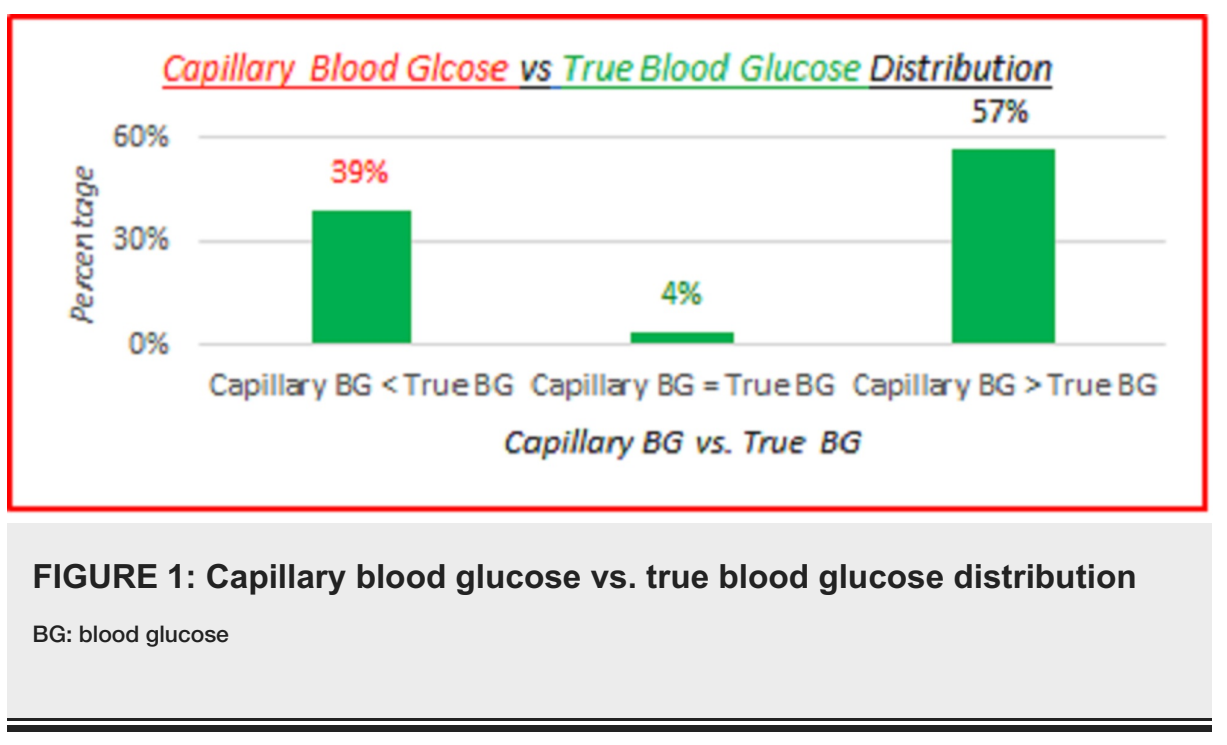

Absolute difference (AD or GAP) (mg/dL) distribution

The data distribution of the absolute difference (AD or GAP) (mg/dL) is shown below in Figure 2. 


\section{Cureus}

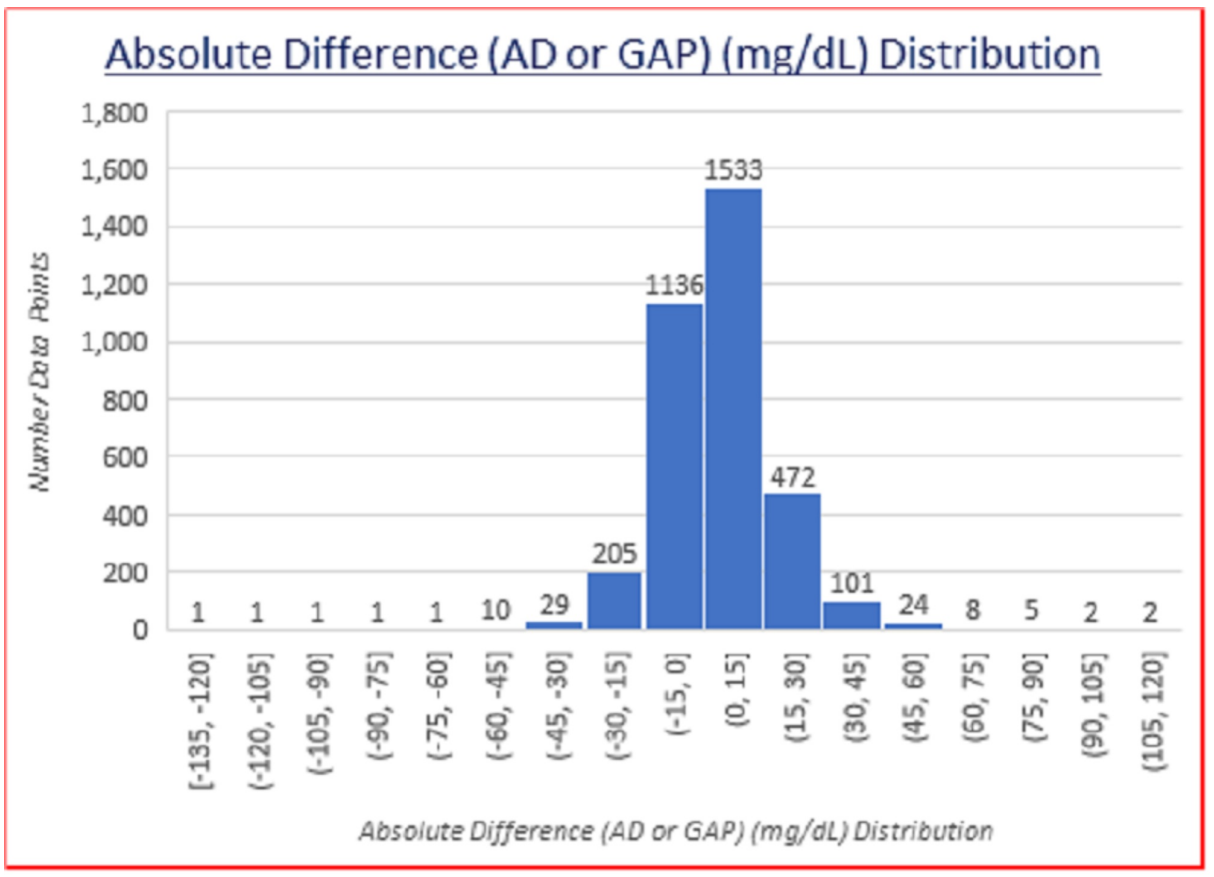

FIGURE 2: Absolute difference (AD or GAP) (mg/dL) distribution

Capillary BG vs. true BG ( $\mathrm{mg} / \mathrm{dL})$ absolute difference (AD or GAP) of unacceptable GAP of $50 \mathrm{mg} / \mathrm{dL}$ and over

The data distribution of the capillary BG vs. true BG (mg/dL) absolute difference (AD or GAP) of unacceptable GAP of $50 \mathrm{mg} / \mathrm{dL}$ and over is shown below in Figure 3. These could result in incorrect medication. The largest GAP was a triple-digit $118 \mathrm{mg} / \mathrm{dL}$.

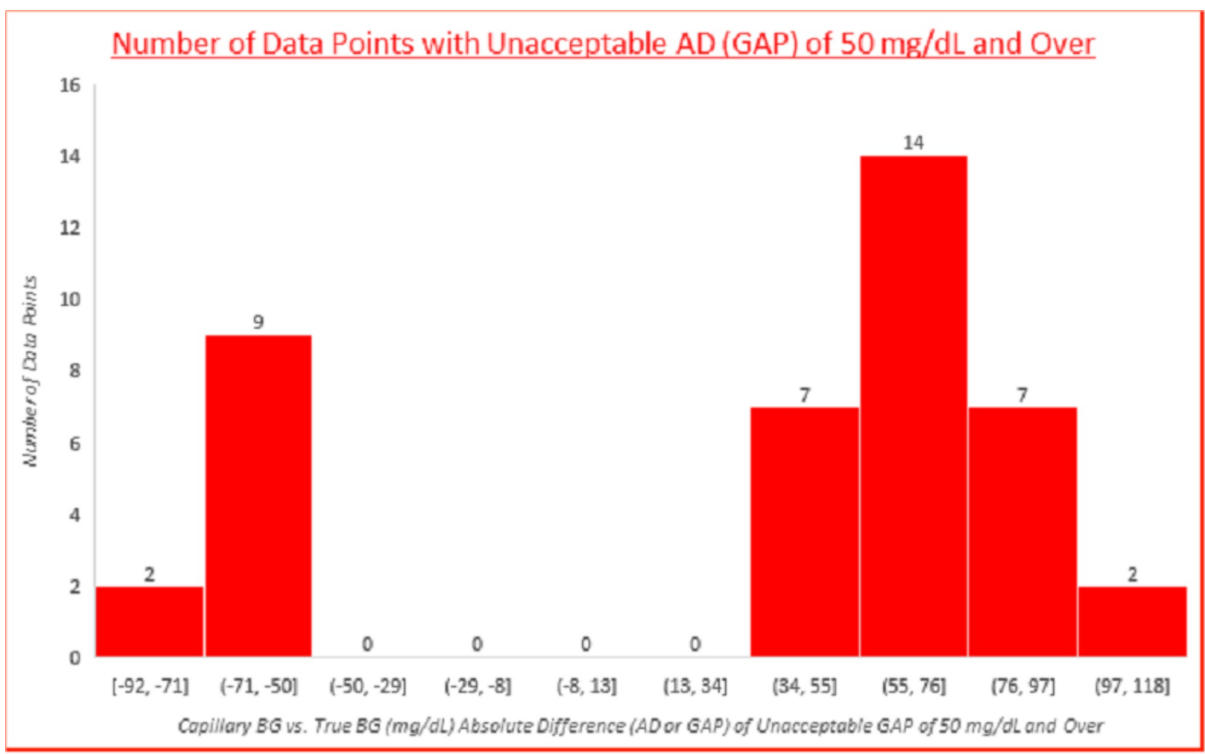

FIGURE 3: Capillary BG vs. true BG (mg/dL) absolute difference (AD or GAP) of unacceptable GAP of $50 \mathrm{mg} / \mathrm{dL}$ and over BG: blood glucose

Absolute relative difference (ARD) (\%) and absolute difference (AD or GAP) (mg/dL) coefficients of variation (CV) (\%)

Absolute relative difference (ARD) (\%) and absolute difference (AD or GAP) (mg/dL) coefficients of variation 


\section{Cureus}

(\%) of $100 \%$ indicate high variance between the capillary BG and venous true BG data. The coefficient of variation is defined as the standard deviation divided by the mean. These are displayed in the data table shown below in Table 2 .

\begin{tabular}{|c|c|c|c|c|c|c|}
\hline \# Data pts & SMBG Device & MARD (\%) & ARD CV (\%) & MAD (mg/dL) & AD CV (\%) & \\
\hline 3,532 & All & $8.3 \%$ & $104 \%$ & 10.9 & $102 \%$ & high variance \\
\hline \multicolumn{7}{|c|}{$\begin{array}{l}\text { TABLE 2: Absolute relative difference }(\%) \text { and absolute difference (AD or GAP) (mg/dL) } \\
\text { coefficients of variation (\%) }\end{array}$} \\
\hline \multicolumn{7}{|c|}{$\begin{array}{l}\text { SMBG: self-monitoring blood glucose; ARD: absolute relative difference; MARD: mean ARD; AD: absolute difference; MAD: mean absolute } \\
\text { difference; CV: coefficient of variation }\end{array}$} \\
\hline
\end{tabular}

\section{SMBG devices (glucometer)}

Five SMBG devices were utilized: one SMBG device for each of three brands, and two generations of SMBG devices for the fourth brand (Device M). Data parameterized according to SMBG devices (glucometer) are fairly consistent. ARD (\%) and absolute difference (AD or GAP) (mg/dL) coefficients of variation (\%) of $100 \%$ indicate high variance between the capillary BG and venous true BG data. These are displayed in the data table and charts below in Table 3 and Figures 4-5.

\begin{tabular}{|c|c|c|c|c|c|}
\hline \# Data pts & SMBG Device & MARD (\%) & ARD CV (\%) & MAD (mg/dL) & AD CV (\%) \\
\hline 3,532 & All & $8.3 \%$ & $104 \%$ & 10.9 & $102 \%$ \\
\hline 244 & Device A & $9.7 \%$ & $113 \%$ & 15.0 & $101 \%$ \\
\hline 1,257 & Device M & $7.0 \%$ & $97 \%$ & 9.4 & $94 \%$ \\
\hline 687 & Device N & $6.5 \%$ & $85 \%$ & 8.7 & $105 \%$ \\
\hline 1,344 & Device O & $10.2 \%$ & $102 \%$ & 12.7 & $98 \%$ \\
\hline
\end{tabular}

TABLE 3: SMBG devices (glucometer), absolute relative difference (ARD) (\%) and absolute difference (AD or GAP) (mg/dL) coefficients of variation (\%)

SMBG: self-monitoring blood glucose; ARD: absolute relative difference; MARD: mean absolute relative difference; AD: absolute difference; MAD: mean absolute difference; CV: coefficient of variation

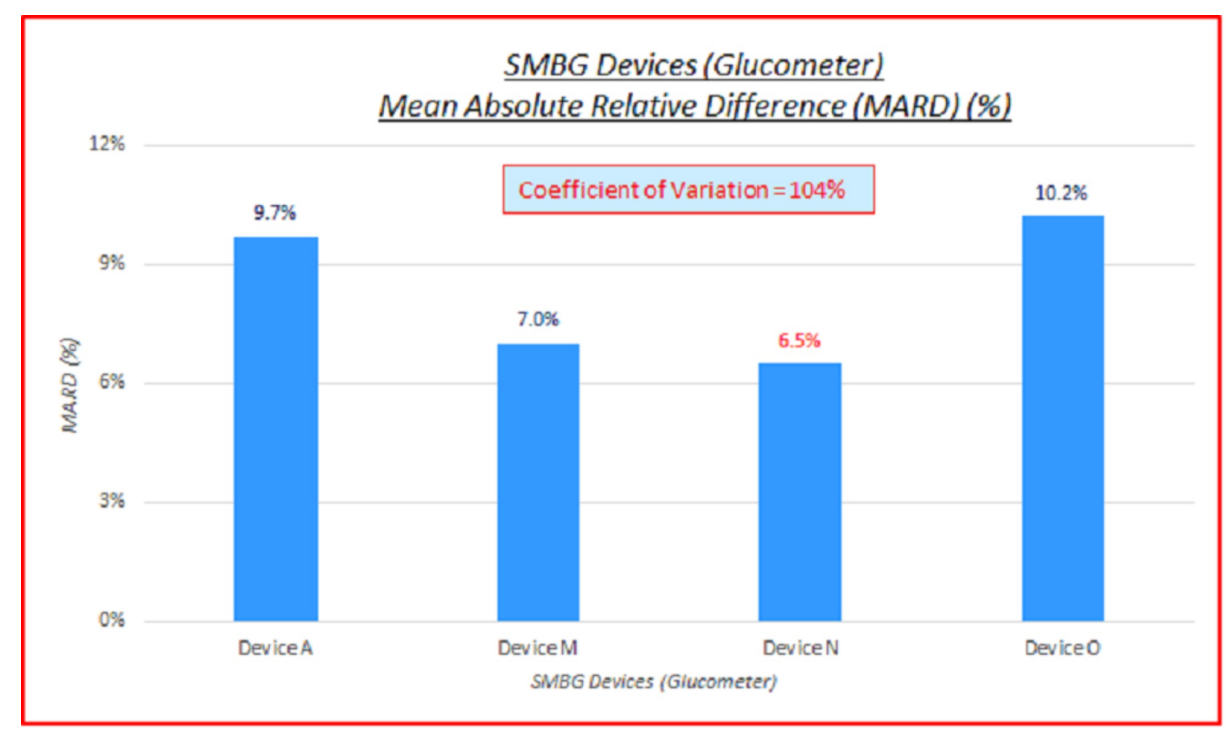

FIGURE 4: SMBG devices (glucometer) MARD (\%) 


\section{Cureus}

SMBG: self-monitoring blood glucose, MARD: mean absolute relative difference

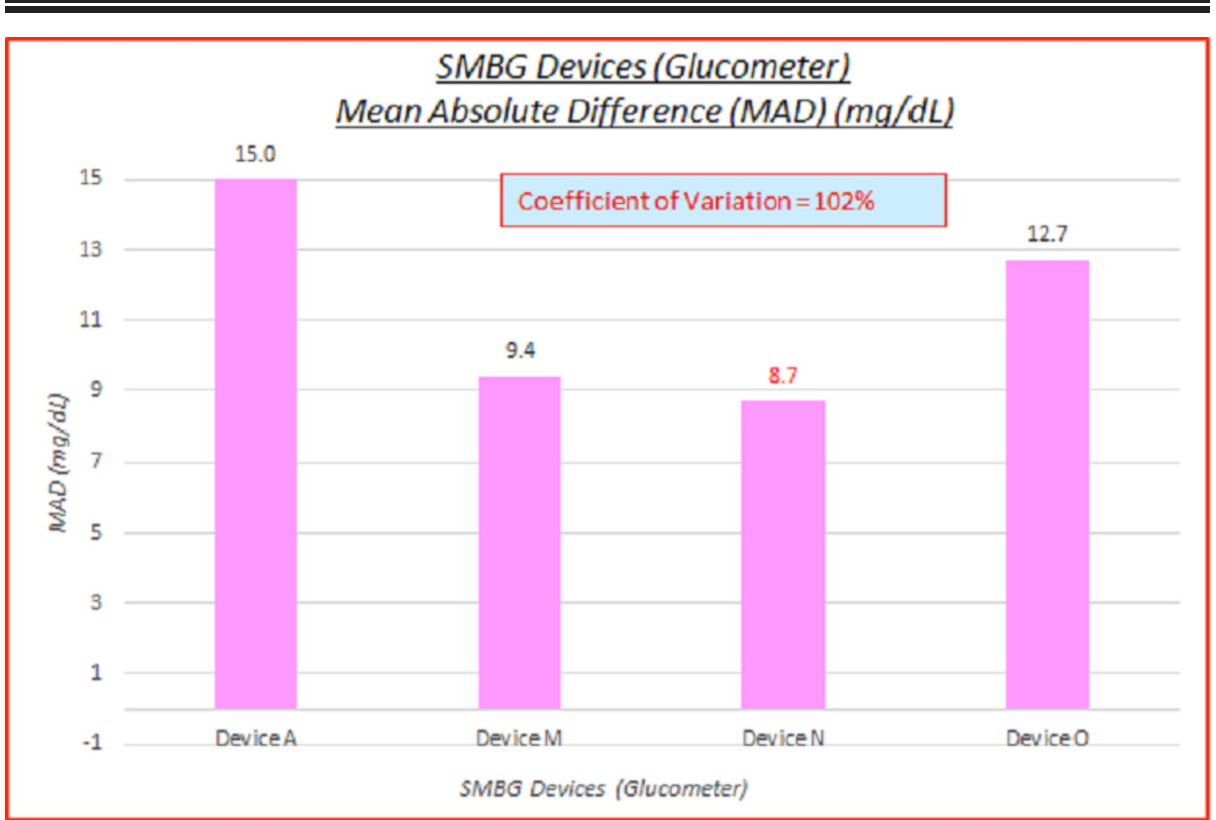

FIGURE 5: SMBG devices (glucometer) mean absolute difference (MAD)

(\%)

SMBG: self-monitoring blood glucose; MAD: mean absolute difference

\section{Body mass index (BMI)}

Data parameterized according to BMI $\left(\mathrm{kg} / \mathrm{m}^{2}\right.$ ) are fairly consistent. ARD (\%) and absolute difference (AD or $\mathrm{GAP})(\mathrm{mg} / \mathrm{dL}$ ) coefficients of variation (\%) of $100 \%$ indicate high variance between the capillary BG and venous true BG data. They show a slight skewing of mean absolute relative difference (MARD) and mean absolute difference (MAD) to the lower BMI side. These are displayed in the data table and charts below in Table 4 and Figures 6-7.

\begin{tabular}{|c|c|c|c|c|c|}
\hline \# Data points & BMI $\left(\mathrm{kg} / \mathrm{m}^{2}\right)$ & MARD (\%) & ARD CV (\%) & MAD (mg/dL) & AD CV (\%) \\
\hline 3,532 & All & $8.3 \%$ & $104 \%$ & 10.9 & $102 \%$ \\
\hline 485 & $<20$ & $9.6 \%$ & $100 \%$ & 13.1 & $95 \%$ \\
\hline 1,425 & $20-<24$ & $9.1 \%$ & $111 \%$ & 11.2 & $101 \%$ \\
\hline 1,261 & $24-28$ & $7.2 \%$ & $92 \%$ & 9.6 & $104 \%$ \\
\hline 325 & $>28$ & $7.9 \%$ & $96 \%$ & 11.9 & $98 \%$ \\
\hline 36 & N/A & & & & \\
\hline
\end{tabular}

TABLE 4: Body mass index (BMI), absolute relative difference (ARD) (\%), absolute difference (AD or GAP) (mg/dL), coefficients of variation (\%)

ARD: absolute relative difference; MARD: mean absolute relative difference; AD: absolute difference; MAD: mean absolute difference, CV: coefficient of variation 


\section{Cureus}

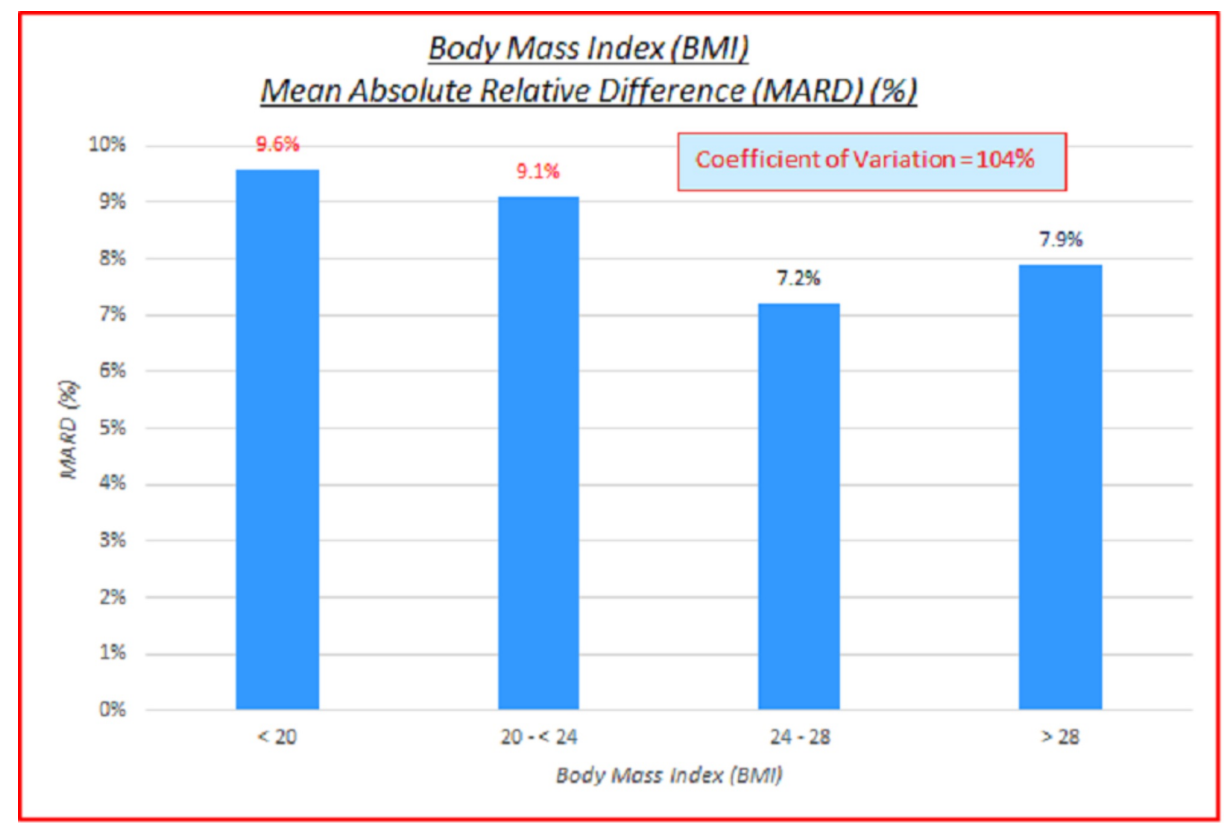

FIGURE 6: Body mass index (BMI) mean absolute relative difference (MARD) (\%)

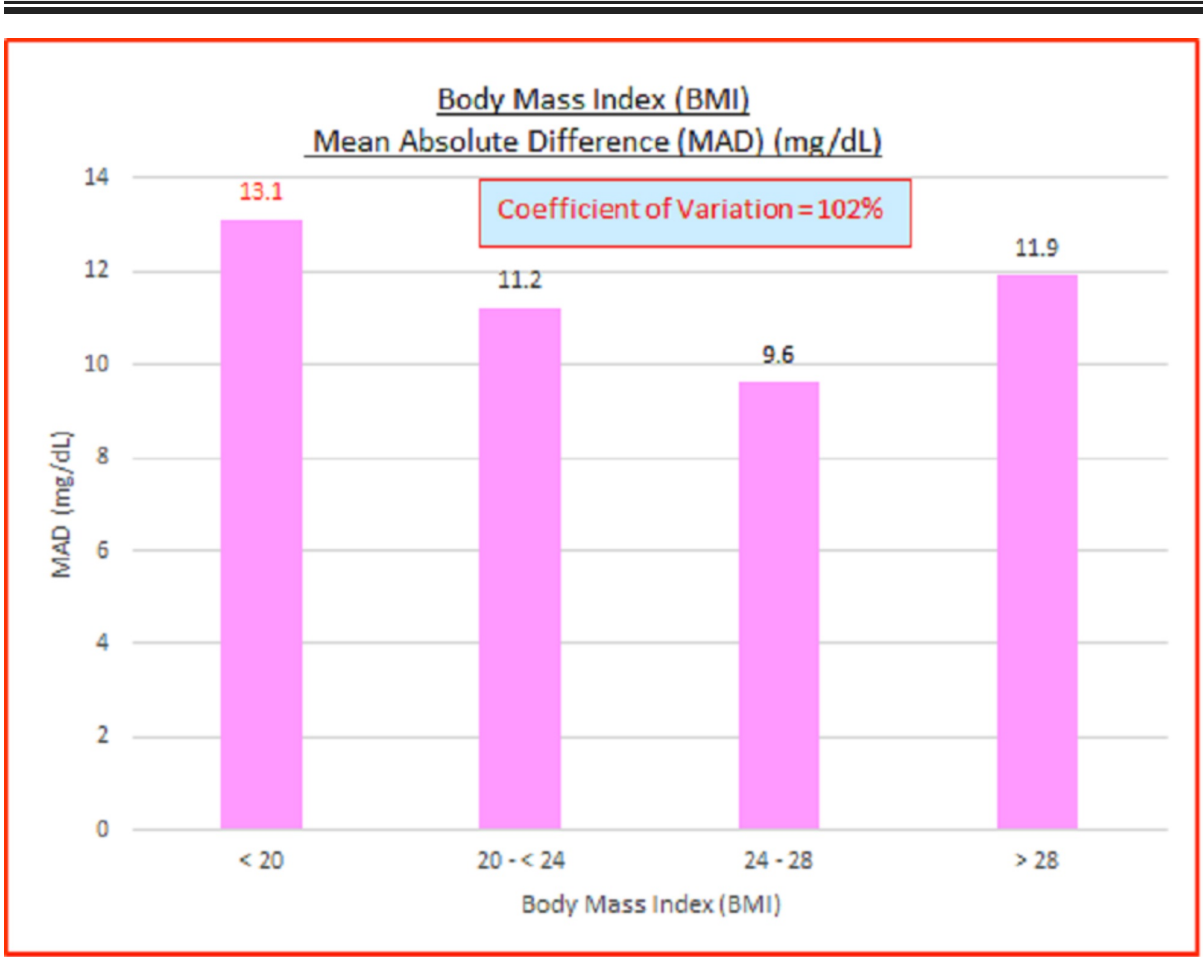

FIGURE 7: Body mass index (BMI) mean absolute difference (MAD)

\section{Age}

Data parameterized according to age are fairly consistent. ARD (\%) and absolute difference (AD or GAP) $(\mathrm{mg} / \mathrm{dL})$ Coefficients of variation (\%) of $100 \%$ indicate high variance between the capillary BG and venous true BG data. They show a slight skewing of MARD and mean MAD to the higher age side. These are displayed in the data table and charts shown below in Table 5 and Figures 8-9. 


\section{Cureus}

\begin{tabular}{|c|c|c|c|c|c|}
\hline \# Data pts & Age & MARD (\%) & ARD CV (\%) & MAD (mg/dL) & AD CV (\%) \\
\hline 3,532 & All & $8.3 \%$ & $104 \%$ & 10.9 & $102 \%$ \\
\hline 413 & $<60$ & $7.4 \%$ & $105 \%$ & 10.6 & $102 \%$ \\
\hline 934 & $60-69$ & $7.2 \%$ & $85 \%$ & 10.3 & $92 \%$ \\
\hline 1,346 & $70-79$ & $8.3 \%$ & $101 \%$ & 10.6 & $104 \%$ \\
\hline 839 & $80+$ & $10.1 \%$ & $112 \%$ & 12.2 & $104 \%$ \\
\hline
\end{tabular}

TABLE 5: Age absolute relative difference (\%) and absolute difference (AD or GAP) (mg/dL) coefficients of variation (\%)

ARD: absolute relative difference; MARD: mean ARD; AD: absolute difference; MAD: mean AD; CV: coefficient of variation

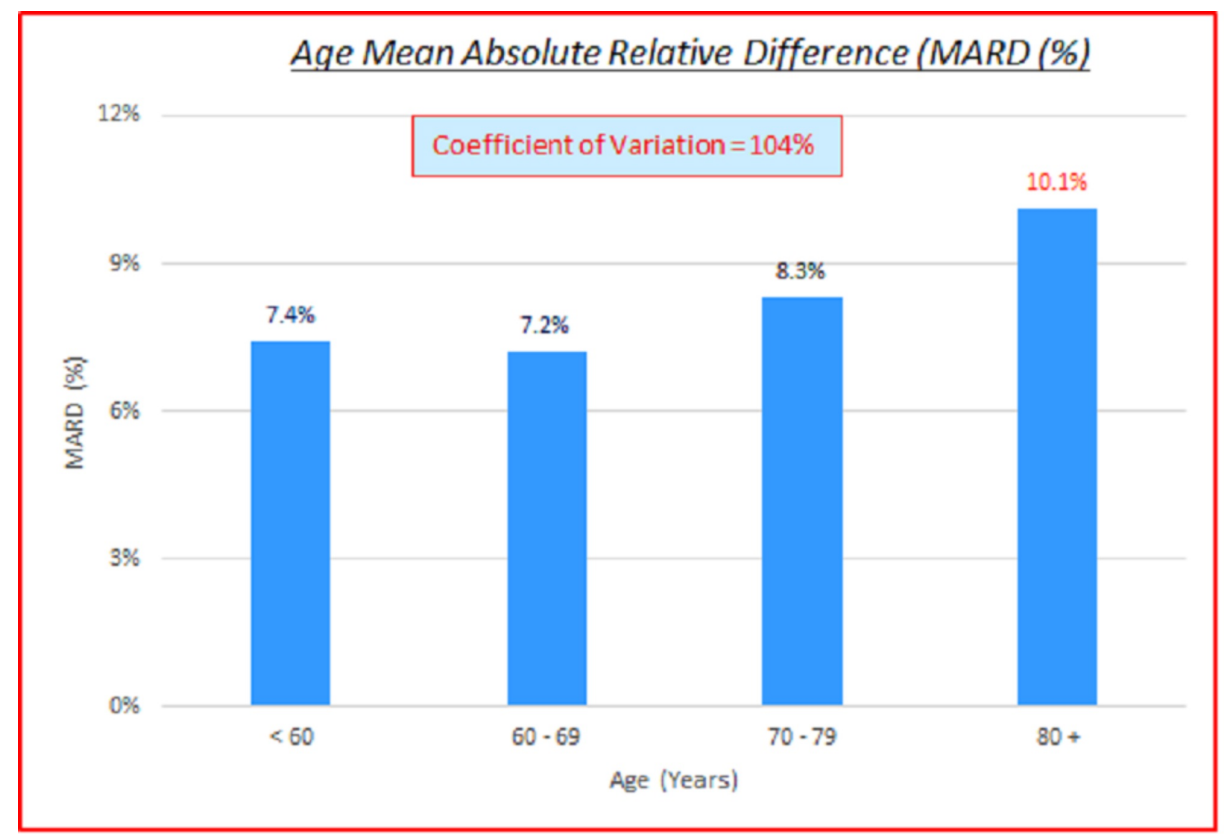

FIGURE 8: Age MARD (\%)

MARD: mean absolute relative difference 


\section{Cureus}

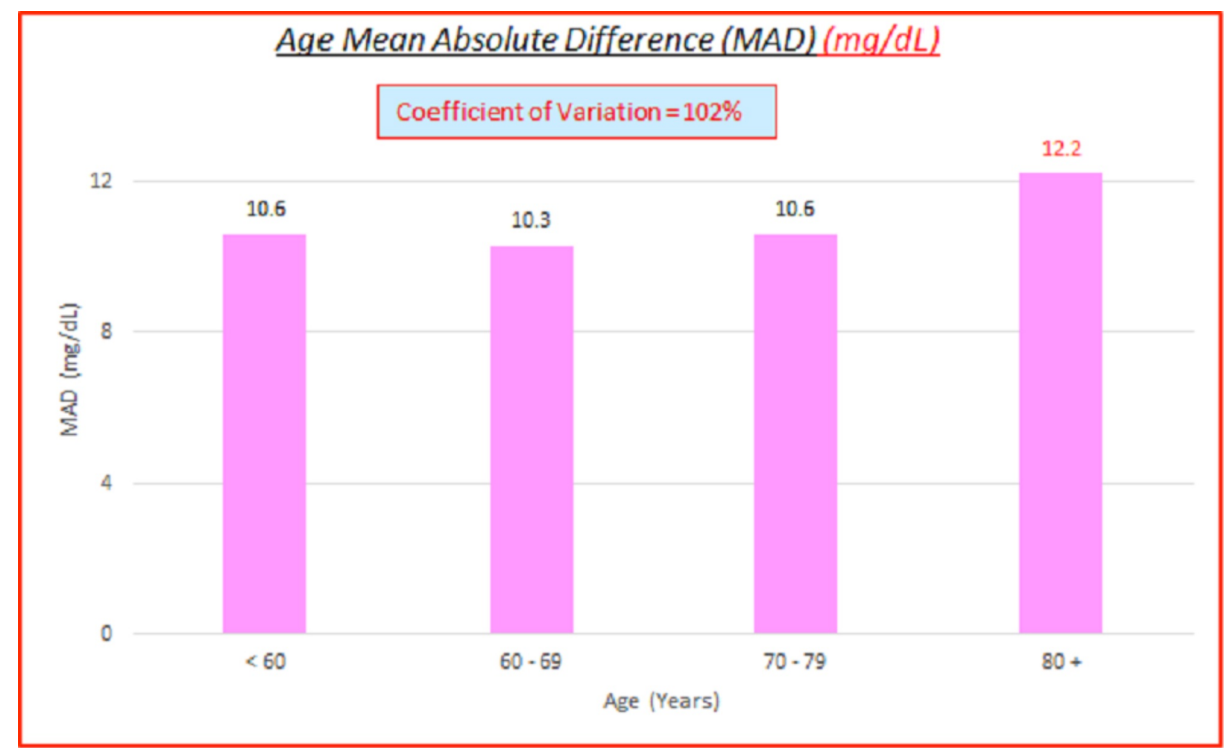

FIGURE 9: Age MAD

MAD: mean absolute difference

\section{Gender}

Data parameterized according to gender are fairly consistent. ARD (\%) and absolute difference (AD or GAP) $(\mathrm{mg} / \mathrm{dL})$ coefficients of variation (\%) of $100 \%$ indicate high variance between the capillary BG and venous true BG data. They show a slight skewing of MARD and MAD to the female gender side. These are displayed in the data table and charts shown below in Table 6 and Figures 10-11.

\begin{tabular}{|c|c|c|c|c|c|}
\hline \# Data pts & Gender & MARD (\%) & ARD CV (\%) & MAD (mg/dL) & AD CV (\%) \\
\hline 3,532 & All & $8.3 \%$ & $104 \%$ & 10.9 & $102 \%$ \\
\hline 2,360 & M & $8.0 \%$ & $103 \%$ & 10.7 & $102 \%$ \\
\hline 1,172 & $F$ & $8.9 \%$ & $105 \%$ & 11.2 & $102 \%$ \\
\hline
\end{tabular}

TABLE 6: Gender absolute relative difference (ARD) (\%) and absolute difference (AD or GAP) $(\mathrm{mg} / \mathrm{dL})$ coefficients of variation (\%)

MARD: absolute relative difference; AD: absolute difference; MAD denotes mean absolute difference, $\mathrm{CV}$ : coefficient of variation 


\section{Cureus}

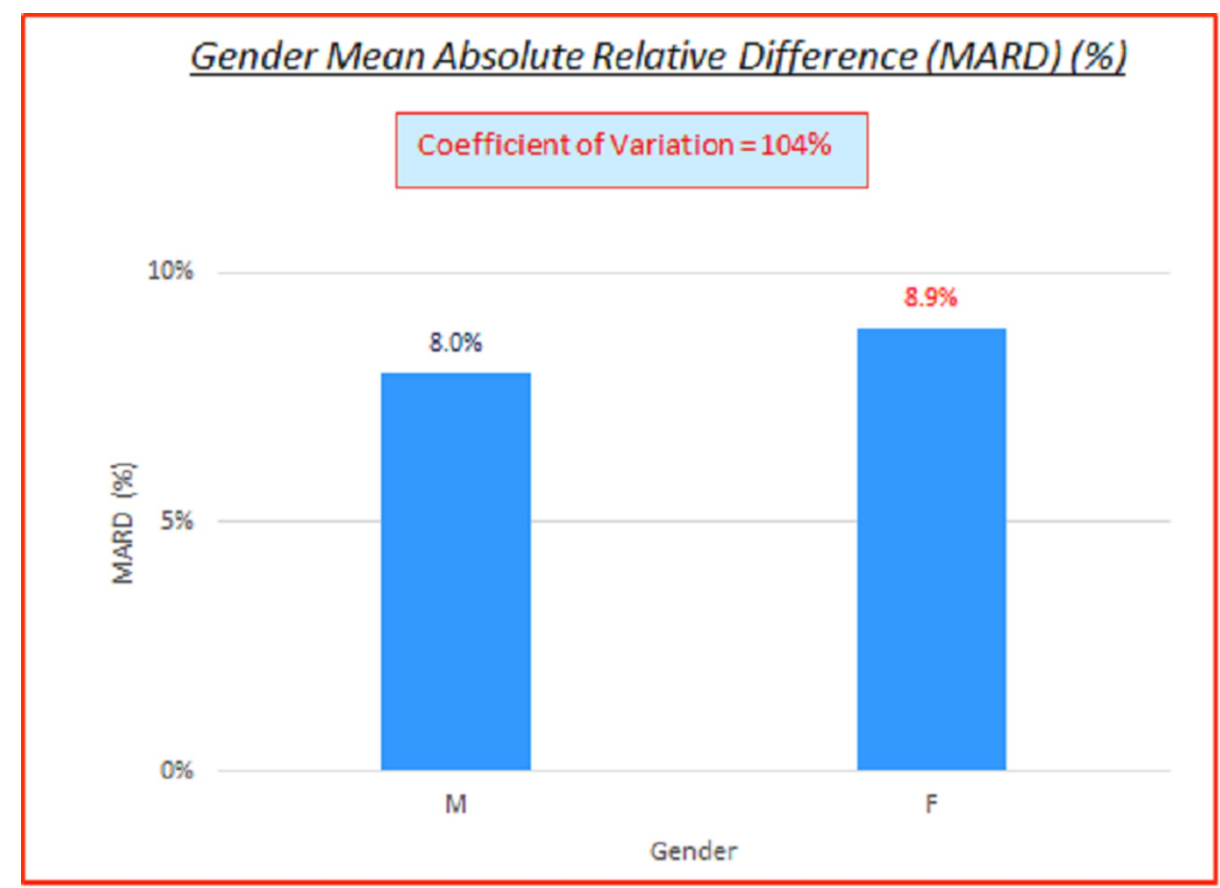

FIGURE 10: Gender MARD (\%)

MARD: mean absolute relative difference

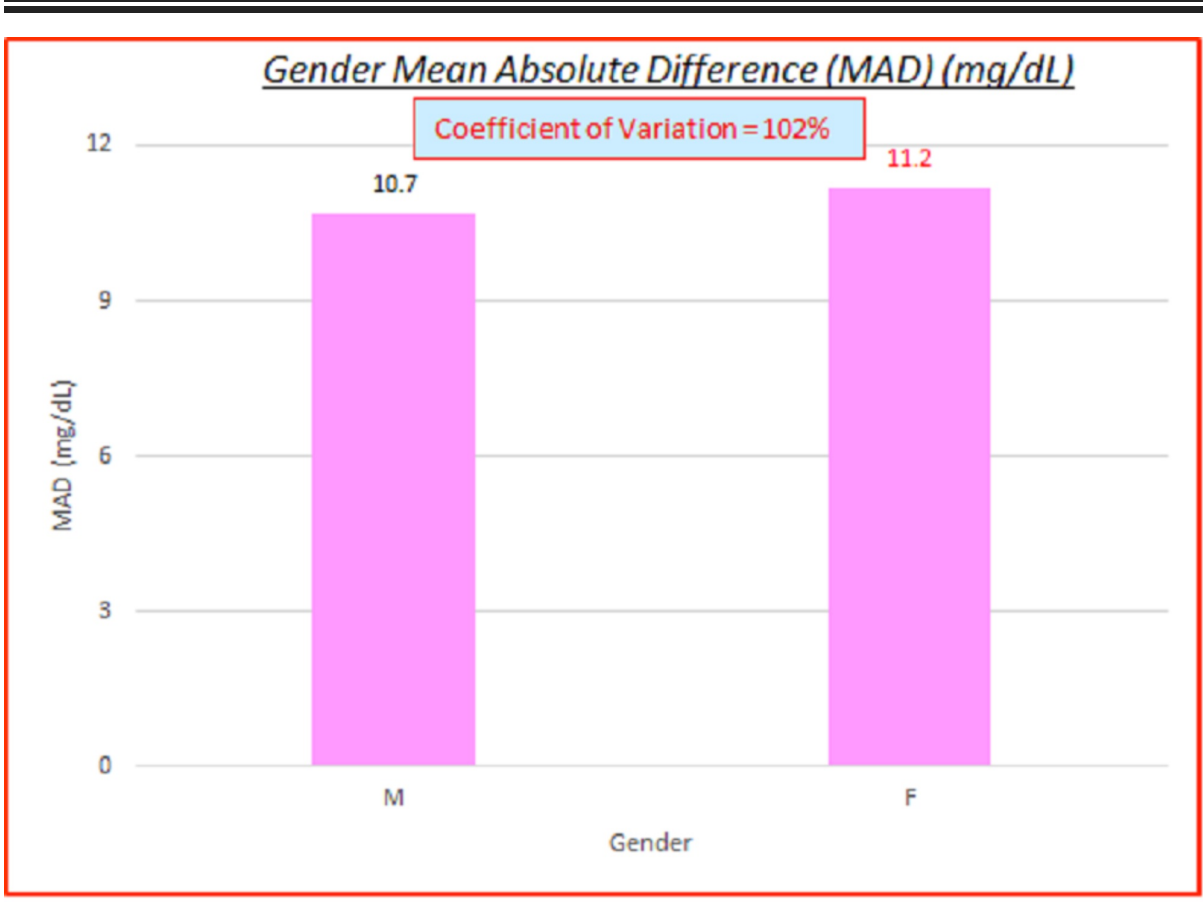

FIGURE 11: Gender MAD (mg/dL)

MAD: mean absolute difference

\section{Discussion}

SMBG devices under-estimated true BG 39\% of the time, and over-estimated true BG $57 \%$ of the time. Overall MARD was $8.3 \%$, and overall MAD was $10.9 \mathrm{mg} / \mathrm{dL}$. Coefficients of variation of $100 \%$ or over indicate high variance between the capillary BG and true BG data. There wasn't any significant difference between the SMBG devices. There was a slight skewing of MARD and MAD to the lower BMI side, the higher age side, and the female side. 
SMBG devices have been on the market for over 30 years, and they have been improved over time. Although they were designed for patients to use at home, they are also used frequently in hospitals, emergency rooms, patients' wards, physicians' offices, and senior homes for quick and convenient checking of blood glucose level.

One of the most critical uses and applications is to evaluate coma patients. Therefore, healthcare providers should be duly apprised and aware that there is a certain GAP between SMBG device measurements and true BG in order to avoid inappropriate and erroneous administration of dextrose or insulin.

There are several studies comparing SMBG device measurement and venous blood laboratory data [5-10]. Boyd et al. compared capillary and venous blood using glucometer and laboratory analyzer, and the mean difference was $0.58 \mathrm{mmol} / \mathrm{L}(10.4 \mathrm{mg} / \mathrm{dL})$ [5]. Sato et al. also compared SMBG devices and venous blood laboratory tests, and revealed that the mean absolute difference was $10.2 \mathrm{mg} / \mathrm{dL}$, and MARD was $7.2 \%$ [6]. The MARDs and MADs of our extensive research are comparable to their data, but our research has far more data points over far longer follow-up periods of time.

The International Organization for Standardization (ISO) recommends that total analytical error for a glucometer be within $\pm 15 \mathrm{mg} / \mathrm{dL}$ when laboratory glucose values are $<100 \mathrm{mg} / \mathrm{dL}$; the acceptable error should be within $15 \%$ for laboratory values $\geqq 100 \mathrm{mg} / \mathrm{dL}[11]$. Thus, it is not at all unexpected nor at all surprising that the overall reported MARDs and MADs were consistent with this because the SMBG devices which were used in the previous researches as well as in our study have already met the criteria before being allowed on the market. However, the extensive database underlying our research revealed large erroneous variations among the data. Absolute Differences were observed over $50 \mathrm{mg} / \mathrm{dL}$ which are clearly not acceptable for medical practice.

There were 41 data points with gaps of and over $50 \mathrm{mg} / \mathrm{dL}$. The largest gap was a triple-digit $118 \mathrm{mg} / \mathrm{dL}$ with a capillary BG of $422 \mathrm{mg} / \mathrm{dL}$ and true BG of 304. If this was in a hospital emergency room, the patient would have been administered inappropriately excessive amounts of insulin. Another case data point showed a capillary BG of $37 \mathrm{mg} / \mathrm{dL}$ and true BG for a gap of $116 \mathrm{mg} / \mathrm{dL}$, and this patient was immediately given dextrose due to the low BG reading of the SMBG device which turned out to be incorrect as shown by the laboratory true BG data several days later. This huge gap caused inappropriate medication which could have been dangerous.

Ginsberg BH wrote in his review that glucose monitoring has some limitations regarding accuracy due to strip factors, environment factors, patient factors, and exogenous interfering substances [12]. The Committee on Standardization of Laboratory Testing Related to Diabetes Mellitus reported that there are significant device-to-device variations, and that while the devices available in Japan met the ISO/TC 212 requirements, adjustments or conversions were added to display data [13]. The committee declares that it is generally known that there is a difference between finger-pricked capillary blood glucose level and venous blood glucose level. Thus, there are many factors causing the gap between capillary BG and true BG.

All healthcare providers should therefore be keenly aware that there are certain gaps between SMBG device measurement and true BG, which could be dangerous. Although SMBG devices are convenient, caution must be duly exercised in accepting the results in medical practice, and particularly to senior female patients with low BMI.

\section{Conclusions}

There is thus shown certain gaps between SMBG device measurements and true BG. Even though MARDs and/or MADs may meet certain criterions of accuracy, the devices themselves do indeed have certain inherent significant errors which can cause wrong medication and/or treatment that could be dangerous, particularly in the use of SMBG devices in the home where most testing is performed.

\section{Additional Information \\ Disclosures}

Human subjects: Consent was obtained by all participants in this study. Animal subjects: All authors have confirmed that this study did not involve animal subjects or tissue. Conflicts of interest: In compliance with the ICMJE uniform disclosure form, all authors declare the following: Payment/services info: All authors have declared that no financial support was received from any organization for the submitted work. Financial relationships: All authors have declared that they have no financial relationships at present or within the previous three years with any organizations that might have an interest in the submitted work. Other relationships: All authors have declared that there are no other relationships or activities that could appear to have influenced the submitted work.

\section{Acknowledgements}

The authors wish to thank Mr. Satoshi John Harada for providing technical support analyzing data. 


\section{References}

1. Rydén L, Grant PJ, Anker SD, et al.: ESC Guidelines on diabetes, pre-diabetes, and cardiovascular diseases developed in collaboration with the EASD: the Task Force on diabetes, pre-diabetes, and cardiovascular diseases of the European Society of Cardiology (ESC) and developed in collaboration with the European Association for the Study of Diabetes (EASD). Eur Heart J. 2013, 34:3035-3087. 10.1093/eurheartj/eht108

2. American Diabetes Association: Standards of medical care in diabetes-2013. Diabetes Care. 2013, 36:S11S66. 10.2337/dc13-S011

3. Clark LD, Lyons C: Electrode systems for continuous monitoring in cardiovascular surgery . Ann N Y Acad Sci. 1962, 102:29-45. 10.1111/j.1749-6632.1962.tb13623.x

4. Toyama S: History and current state of development of blood glucose meter [article in Japanese] . Bulletin of the National Rehabilitation Center for Persons with Disabilities. 2010, 31:1-8.

5. Boyd R, Leigh B, Stuart P: Capillary versus venous bedside blood glucose estimations . Emerg Med J. 2005, 22:177-179. 10.1136/emj.2003.011619

6. Sato T, Oshima H, Nakata K, et al.: Accuracy of flash glucose monitoring in insulin-treated patients with type 2 diabetes. J Diabetes Investig. 2019, 10:846-850. 10.1111/jdi.12954

7. Sylvain HF, Pokorny ME, English SM, et al.: Accuracy of fingerstick glucose values in shock patients . Am J Crit Care. 1995, 4:44-48.

8. Thomas SH, Gough JE, Benson N, Austin PE, Stone CK: Accuracy of fingerstick glucose determination in patients receiving CPR. South Med J. 1994, 87:1072-1075. 10.1097/00007611-199411000-00003

9. Adnan M, Imam F, Shabbir I, Ali Z, Rahat T: Correlation between capillary and venous blood glucose levels in diabetic patients. Asian Biomed (Res Rev News). 2015, 9:55-59. 10.5372/1905-7415.0901.368

10. Yang C, Chang C, Lin J: A comparison between venous and finger-prick blood sampling on values of blood glucose. Int Proc Chem Biol Environ Eng. 2012, 39:206-210.

11. Jendrike N, Baumstark A, Kamecke U, Haug C, Freckmann G: ISO 15197: 2013 Evaluation of a blood glucose monitoring system's measurement accuracy. J Diabetes Sci Technol. 2017, 11:1275-1276. $10.1177 / 1932296817727550$

12. Ginsberg BH: Factors affecting blood glucose monitoring: sources of errors in measurement . J Diabetes Sci Technol. 2009, 3:903-913. 10.1177/193229680900300438

13. Tominaga M, Kobayashi I, Kuwa K, et al.: Report of the committee on standardization of laboratory testing related to diabetes mellitus: standardization and proper use of devices for self-monitoring of blood glucose [article in Japanese]. Journal of the Japan Diabetic Society. 2001, 44:165-176. 